that Congress should "consider a fundamental re-examination of the system".

The alarm sounded by the GAO is likely to resonate with the public, after a three-month stretch last autumn in which outbreaks of foodborne disease killed three people and made more than 500 others sick. The culprits ranged from spinach contaminated with the bacterium Escherichia coli, to salmonella-bearing tomatoes, to lettuce that probably infected scores of people with E. coli after they ate at Taco Bell and Taco John's restaurants.

These well-publicized incidents stand out against a far larger, latent problem. According to the Centers for Disease Control and Prevention in Atlanta, 76 million Americans contract food-borne illnesses each year, and 5,000 of them die.

A science-backed regulatory system is needed to address an issue on this scale. What has evolved instead over the past century is an irrational and expensive arrangement, whereby officials examine every carcass at every slaughterhouse in the United States every day, but a major food processing plant may escape inspection for a decade.

At the same time, the government research programmes supporting food-safety regulation are neither comprehensive nor coordinated. Research is scattered between often-obscure subdivisions of several departments, from the Environmental Protection Agency to the US Department of Agriculture (USDA). The result is a patchwork of research that, according to a 1998 Institute of Medicine report that reads uncannily like the GAO's latest assessment, "raises serious concerns about duplication of effort and about the linkage of science to attempt to solve food safety problems of the highest priority".

For historical reasons, the USDA is responsible for the safety of meat, poultry and processed egg products, whereas most of the rest $-80 \%$ of the food supply - rests with the Food and Drug Administration (FDA).

But the FDA's Center for Food Safety and Applied Nutrition in Col- lege Park, Maryland, which bears the primary responsibility for the research that supports food safety at the agency, has seen its budget and staff cut over the past decade. This year, its operating budget will fall to $\$ 25$ million - a little over half of what it was in 2003 . The centre has far less funding for its own science, and next to none for extramural research in areas it needs to learn more about, from microbial ecology to detection methodologies for pathogens in food.

The recent outbreaks caused by bacteria in fresh fruit and vegetables illustrate the paucity of research.

Nine years ago, after a string of comparable outbreaks, the FDA issued a set of general recommendations on managing manure, irrigation water and farmworker hygiene to minimize contamination of fruit and vegetables. But it has not done the research to get the data needed to convert these general guidelines into firm, quantified regulations to be implemented on farms, and during food processing and transportation.

In the meantime, Democrats in Congress have repeatedly introduced legislation to establish a single US food-safety agency. It is tempting to believe that this approach would produce the necessary coherence in food safety. But past experience of amalgamating parts of the US federal government, from the Department of Energy to the Department of Homeland Security, does not give cause for optimism that such a consolidation would be either efficient or effective.

The best approach may instead involve three more modest steps: an inter-agency panel to properly coordinate food safety; a comprehensive revision of the antiquated and fragmented legislation now governing it, to better reflect today's risks and today's science; and a properly supported, coordinated research programme to inform food safety policy and practice.

\title{
Methods in full
}

\section{From now on, Nature authors will be able to include more experimental details in their papers.}

W

hen in 1960 Theodor Maiman reported the creation of the laser, he did so in about 300 words. Most of these were about the principles. The experiment was described in two sentences (see Nature 187, 493-494; 1960).

Until now, Nature's style of research papers - although more generous in the space allowed than it once was - has been grounded in this telegraphic tradition, allowing comparatively little space for experimental detail. Consequently, with the advent of the Internet, the supplementary material published online has grown voluminous, and nearly ubiquitous - appended to every Article and Letter in this week's issue, for example. And some of it isn't supplementary at all - it is essential for anyone trying to replicate the work.

We have now taken steps to do better justice to what authors have to say, by letting them present full experimental methods as an integral part of their paper. It is clear that more and more people read papers only in their online versions. So we are expanding the online versions of our Articles and Letters, while condensing some of the technical detail in the printed version.

To be specific: in those papers requiring a separate methods section, the online version of the paper will allow authors to include enough detail to satisfy their peers. This is not a 'supplementary' methods file, but a component of the paper, with all the virtues of full-text linking and functionality. It will appear in all online versions, including the authors' versions of papers that can be loaded into PubMed Central and other open-access repositories six months after publication.

But Nature also rejoices in being a print publication. We have no wish to leave print readers lacking sufficient understanding of what was done to appreciate the authors' achievements. Accordingly, the print version will include a 300 -word summary of the methods. This will also appear in the online version.

Norman Lockyer, the founding editor of Nature, might well deplore the loss of brevity in today's scientific reports. But our authors should bear in mind that readers still value succinctness - and that Nature's editors and copy-editors will continue to insist on it. 